\title{
EQUIVALENCE AND NONEXISTENCE OF STANDING WAVES FOR COUPLED SCHRÖDINGER EQUATIONS WITH CHERN-SIMONS GAUGE FIELDS
}

\author{
Hyunguin Huh - Jinmyoung SeOK
}

\begin{abstract}
This paper is devoted to the study of standing waves for socalled $N=2$ supersymmetric Chern-Simons-Schrödinger equations, a coupled system of Schrödinger equations in which Chern-Simons gauge fields are incorporated. We show that there is no nontrivial standing wave to the $N=2$ supersymmetric Chern-Simons-Schrödinger equations when the coupling constants are less than critical numbers. We also prove that static $N=2$ supersymmetric Chern-Simons-Schrödinger equations are equivalent to their first order self-dual system when the coupling constants are critical.
\end{abstract}

2010 Mathematics Subject Classification. 35L15, 35L45, 35Q40, 35F25.

Key words and phrases. $N=2$ supersymmetric Chern-Simons-Schrödinger, critical coupling constants, self-dual system.

H. Huh was supported by Basic Science Research Program through the National Research Foundation of Korea(NRF) funded by the Ministry of Education (2014R1A1A2053747).

J. Seok was supported by Basic Science Research Program through the National Research Foundation of Korea(NRF) funded by the Ministry of Education (2014R1A1A2054805) and was also supported by the POSCO TJ Park Science Fellowship. 


\section{Introduction}

In this paper, we are concerned with the following coupled Schrödinger equations with the Chern-Simons gauge fields

$$
\begin{aligned}
& i D_{0} \phi+\left(D_{1} D_{1}+D_{2} D_{2}\right) \phi+2 \lambda_{1}|\phi|^{2} \phi+\lambda_{2}|\psi|^{2} \phi,=0, \\
& i D_{0} \psi+\left(D_{1} D_{1}+D_{2} D_{2}\right) \psi+\lambda_{2}|\phi|^{2} \psi-\left(\partial_{1} A_{2}-\partial_{2} A_{1}\right) \psi=0, \\
& \partial_{0} A_{1}-\partial_{1} A_{0}=-\operatorname{Im}\left(\bar{\phi} D_{2} \phi\right)-\operatorname{Im}\left(\bar{\psi} D_{2} \psi\right)+\partial_{1}|\psi|^{2} / 2, \\
& \partial_{0} A_{2}-\partial_{2} A_{0}=\operatorname{Im}\left(\bar{\phi} D_{1} \phi\right)+\operatorname{Im}\left(\bar{\psi} D_{1} \psi\right)+\partial_{2}|\psi|^{2} / 2 \\
& \partial_{1} A_{2}-\partial_{2} A_{1}=-\left(|\phi|^{2}+|\psi|^{2}\right) / 2
\end{aligned}
$$

where $i$ denotes the imaginary unit, $\partial_{0}=\partial / \partial t, \partial_{1}=\partial / \partial x_{1}, \partial_{2}=c \partial / \partial x_{2}$ for $\left(t, x_{1}, x_{2}\right) \in \mathbb{R}^{1+2}, \phi, \psi: \mathbb{R}^{1+2} \rightarrow \mathbb{C}$ are complex scalar fields, $A_{\mu}: \mathbb{R}^{1+2} \rightarrow \mathbb{R}$ are gauge fields, $D_{\mu}=\partial_{\mu}+i A_{\mu}$ are covariant derivative for $\mu=0,1,2$. The parameters $\lambda_{1}, \lambda_{2}>0$ represent the strength of coupling effect of $\phi$ and $\psi$. The system (1.1) is proposed in [7] for explaining electromagnetic phenomena on a planar domain such as fractional quantum Hall effect or high temperature super conductivity. It is called the $N=2$ supersymmetric Chern-Simons-Schrödinger equations because it is $N=2$ supersymmetric extension of the well-known Jackiw-Pi model [6] which may be derived by putting $\psi \equiv 0$ in (1.1).

The aim of this paper is to observe some equivalence and nonexistence results for standing wave solutions to (1.1) in a unified way. We mean, by a standing wave solution, a solution $\left(\phi, \psi, A_{0}, A_{1}, A_{2}\right)$ to (1.1) of the form

$$
\left(\phi(t, x), \psi(t, x), A_{\mu}(t, x)\right)=\left(u(x) \mathrm{e}^{i \omega_{1} t}, v(x) \mathrm{e}^{i \omega_{2} t}, A_{\mu}(x)\right),
$$

where $u, v$ are complex $C^{\infty}$ functions, $A_{\mu}(x)$ are real $C^{\infty}$ functions and $\omega_{1}, \omega_{2}$ are real constants. A standing wave solution with $\omega_{1}=\omega_{2}=0$ is called static.

We first consider a special case where the critical coupling constants $\lambda_{1}$ and $\lambda_{2}$ are critical, i.e. $\lambda_{1}=1 / 2=\lambda_{2}$. In this case, one can derive the following system of first order equations called self dual system:

$$
\begin{aligned}
D_{1} u+i D_{2} u & =0, \\
D_{1} v+i D_{2} v & =0, \\
\partial_{1} A_{2}-\partial_{2} A_{1} & =-\frac{1}{2}\left(|u|^{2}+|v|^{2}\right), \\
A_{0}-\frac{1}{2}|u|^{2} & =0 .
\end{aligned}
$$

We note that solutions of the self-dual equations (1.2) provide static solutions to the equations (1.1) with the critical constants $\lambda_{1}=1 / 2=\lambda_{2}$, i.e. solutions to the following equations

$$
A_{0} u-D_{1} D_{1} u-D_{2} D_{2} u-|u|^{2} u-\frac{1}{2}|v|^{2} u=0
$$




$$
\begin{gathered}
A_{0} v-D_{1} D_{1} v-D_{2} D_{2} v-\frac{1}{2}|u|^{2} v+\left(\partial_{1} A_{2}-\partial_{2} A_{1}\right) v=0, \\
\partial_{1} A_{0}=\operatorname{Im}\left(\bar{u} D_{2} u\right)+\operatorname{Im}\left(\bar{v} D_{2} v\right)-\frac{1}{2} \partial_{1}|v|^{2}, \\
\partial_{2} A_{0}=-\operatorname{Im}\left(\bar{u} D_{1} u\right)-\operatorname{Im}\left(\bar{v} D_{1} v\right)-\frac{1}{2} \partial_{2}|v|^{2}, \\
\partial_{1} A_{2}-\partial_{2} A_{1}=-\frac{1}{2}\left(|u|^{2}+|v|^{2}\right) .
\end{gathered}
$$

Indeed, applying the following identity

$$
\sum_{j=1}^{2} D_{j} D_{j} u=\left(D_{1}-i D_{2}\right)\left(D_{1}+i D_{2}\right) u+\left(\partial_{1} A_{2}-\partial_{2} A_{1}\right) u,
$$

one can check, for instance, the equation (1.3) is verified

$$
A_{0} u-\left(D_{1}-i D_{2}\right)\left(D_{1}+i D_{2}\right) u-\left(\partial_{1} A_{2}-\partial_{2} A_{1}\right) u-|u|^{2} u-\frac{1}{2}|v|^{2} u=0 .
$$

The first main result is to establish the reverse direction. In other words, we prove that every smooth solution of (1.3)-(1.7) with some integrability conditions is a solution of the self-dual system (1.2). This kind of equivalence is proved in [4] for Jackiw-Pi model. Here we extend it to the $N=2$ super-symmetric ChernSimons-Schrödinger equations.

THeorem 1.1. Suppose that $\left(\phi, \psi, A_{0}, A_{1}, A_{2}\right)$ is a static solution of (1.1) such that:

(a) $\phi, \psi \in H^{1}\left(\mathbb{R}^{2}\right)$ and $\phi \not \equiv 0$,

(b) $A_{0} \in L^{p}\left(\mathbb{R}^{2}\right)$ for some $1<p \leq \infty$,

(c) $A_{1}, A_{2} \in L^{q}\left(\mathbb{R}^{2}\right)$ for some $2<q \leq \infty$.

Then $\left(\phi, \psi, A_{0}, A_{1}, A_{2}\right)$ also solves the self dual system (1.2).

Theorem 1.1 says that the system (1.2) equals to the equations (1.3)-(1.7) under the condition of Theorem 1.1 although it consists of first-order differential equations. In the case of $\phi \equiv 0$, we have $A_{0} \psi=0$ and $A_{0}=c$ which implies $A_{0} \equiv 0$ for nontrivial $\psi$. See the proof of Theorem 1.1.

Next, we state nonexistence results of nontrivial standing waves when parameters $\lambda_{1}$ and $\lambda_{2}$ are subcritical, i.e. $\left(\lambda_{1}, \lambda_{2}\right) \in((0,1 / 2] \times(0,1 / 2]) \backslash\{(1 / 2,1 / 2)\}$.

THEOREM 1.2. Suppose that $\left(\phi, \psi, A_{0}, A_{1}, A_{2}\right)$ is a standing wave solution of (1.1) satisfying (a)-(c) of Theorem 1.1 and $0<\lambda_{1}<1 / 2,0<\lambda_{2} \leq 1 / 2$. Then, one has $\phi \equiv 0$. In addition, one also has $\psi \equiv 0$ if $A_{0} \neq-\omega_{2}$.

Theorem 1.3. Suppose that $\left(\phi, \psi, A_{0}, A_{1}, A_{2}\right)$ is a standing wave solution of (1.1) satisfying (a)-(c) of Theorem 1.1 and $\lambda_{1}=1 / 2,0<\lambda_{2}<1 / 2$. Then, one has either $\phi \equiv 0$ or $\psi \equiv 0$. In addition, one has $\psi \equiv 0$ if $A_{0} \neq-\omega_{2}$. 
In fact, Theorems 1.2 and 1.3 are corollaries of Theorems 3.1 and 3.2 in which the equations (2.1), a generalized version of (1.1), is considered. See Section 3. All the theorems mentioned above are derived as easy applications of an integral identity (2.8) which holds true for every standing wave solutions to (2.1). We will see that (2.8) is due to combining Pohozaev type identity and Nehari type identity with Bogomol'nyi trick (2.17) below.

The rest of the paper is organized as follows. We derive (2.8) in Section 2. In sections 3 we give precise statements of Theorems 3.1 and 3.2. In Section 4 we prove Theorems $1.1,3.1$ and 3.2 by using (2.8).

\section{Integral identity}

Consider the generalized version of (1.1) as the following:

$$
\begin{aligned}
& i D_{0} \phi+\left(D_{1} D_{1}+D_{2} D_{2}\right) \phi+F^{\prime}\left(|\phi|^{2}\right) \phi+\frac{\partial G}{\partial s_{1}}\left(|\phi|^{2},|\psi|^{2}\right) \phi=0 \\
& i D_{0} \psi+\left(D_{1} D_{1}+D_{2} D_{2}\right) \psi+\frac{\partial G}{\partial s_{2}} G\left(|\phi|^{2},|\psi|^{2}\right) \psi-\left(\partial_{1} A_{2}-\partial_{2} A_{1}\right) \psi=0 \\
& \partial_{0} A_{1}-\partial_{1} A_{0}=-\operatorname{Im}\left(\bar{\phi} D_{2} \phi\right)-\operatorname{Im}\left(\bar{\psi} D_{2} \psi\right)+\frac{1}{2} \partial_{1}|\psi|^{2} \\
& \partial_{0} A_{2}-\partial_{2} A_{0}=\operatorname{Im}\left(\bar{\phi} D_{1} \phi\right)+\operatorname{Im}\left(\bar{\psi} D_{1} \psi\right)+\frac{1}{2} \partial_{2}|\psi|^{2} \\
& \partial_{1} A_{2}-\partial_{2} A_{1}=-\frac{1}{2}\left(|\phi|^{2}+|\psi|^{2}\right)
\end{aligned}
$$

Throughout the paper, we always assume that $F$ and $G$ are $C^{\infty}$ functions and satisfy

$$
F(0)=F^{\prime}(0)=0 \text { and } G\left(s_{1}, 0\right)=G\left(0, s_{2}\right)=0 \text { for all } s_{1}, s_{2} \geq 0 .
$$

If one choose, for example, $F(s)=\lambda_{1} s^{2}, G\left(s_{1}, s_{2}\right)=\lambda_{2} s_{1} s_{2}$, the system (2.1) becomes (1.1).

Now, we plug the standing wave ansatz into (2.1) to get the equations

$$
\begin{aligned}
\left(\omega_{1}+A_{0}\right) u & -\left(D_{1} D_{1}+D_{2} D_{2}\right) u-F^{\prime}\left(|u|^{2}\right) u-\partial_{s_{1}} G\left(|u|^{2},|v|^{2}\right) u=0 \\
\left(\omega_{2}+A_{0}\right) v & -\left(D_{1} D_{1}+D_{2} D_{2}\right) v \\
& -\partial_{s_{2}} G\left(|u|^{2},|v|^{2}\right) v+\left(\partial_{1} A_{2}-\partial_{2} A_{1}\right) v=0 \\
\partial_{1} A_{0}= & \operatorname{Im}\left(\bar{u} D_{2} u\right)+\operatorname{Im}\left(\bar{v} D_{2} v\right)-\frac{1}{2} \partial_{1}|v|^{2} \\
\partial_{2} A_{0}= & -\operatorname{Im}\left(\bar{u} D_{1} u\right)-\operatorname{Im}\left(\bar{v} D_{1} v\right)-\frac{1}{2} \partial_{2}|v|^{2} \\
\partial_{1} A_{2}-\partial_{2} A_{1}= & -\frac{1}{2}\left(|u|^{2}+|v|^{2}\right)
\end{aligned}
$$

Then, on can obtain the following: 
Proposition 2.1. Let $\left(u, v, A_{0}, A_{1}, A_{2}\right)$ be a smooth solution of equations (2.3)-(2.7). Suppose that

(a) $u, v \in H^{1}\left(\mathbb{R}^{2}\right)$,

(b) $A_{0} \in L^{p}\left(\mathbb{R}^{2}\right)$ for some $1<p \leq \infty$,

(c) $A_{1}, A_{2} \in L^{q}\left(\mathbb{R}^{2}\right)$ for some $2<q \leq \infty$,

(d) $F\left(|u|^{2}\right), F^{\prime}\left(|u|^{2}\right)|u|^{2}, G\left(|u|^{2},|v|^{2}\right), \partial_{s_{1}} G\left(|u|^{2},|v|^{2}\right)|v|^{2}, \partial_{s_{2}} G\left(|u|^{2},|v|^{2}\right)|v|^{2}$ in $L^{1}\left(\mathbb{R}^{2}\right)$.

Then the following integral identity holds true:

$$
\begin{aligned}
\int_{\mathbb{R}^{2}}\left|\left(D_{1}+i D_{2}\right) u\right|^{2} & +\left|\left(D_{1}+i D_{2}\right) v\right|^{2}+\frac{1}{2}|u|^{4} \\
+F\left(|u|^{2}\right)- & F^{\prime}\left(|u|^{2}\right)|u|^{2}+\frac{1}{2}|u|^{2}|v|^{2}+G\left(|u|^{2},|v|^{2}\right) \\
& \quad-\partial_{s_{1}} G\left(|u|^{2},|v|^{2}\right)|u|^{2}-\partial_{s_{2}} G\left(|u|^{2},|v|^{2}\right)|v|^{2} d x=0 .
\end{aligned}
$$

Here, $H^{1}\left(\mathbb{R}^{2}\right)$ denotes the standard Sobolev space which is the set of weakly differentiable functions $u$ on $\mathbb{R}^{2}$ such that both of $u$ and $\nabla u$ are square integrable. We will derive the identity (2.8) by combining the well-known Derrick-Pohozaev type identity [2] and Nehari type identity with the Bogomol'nyi trick (2.17).

Proof. The main part of the proof is to multiply (2.3) and (2.4) by $x_{k} \overline{D_{k} u}$ and $x_{k} \overline{D_{k} v}$ respectively and to integrate them to derive the Derrick-Pohozaev identity. Then the Bogomol'nyi trick (2.17) is used to obtain (2.8). Rigorous proof consists of integrating by parts over $B_{R}:=\left\{x \in \mathbb{R}^{2}|| x \mid \leq R\right\}$, taking care of boundary terms and applying the Lebesgue dominated convergence theorem as in [4]. Here we skip a detailed process and just show main calculation for simple presentation. From now on, we adopt the summation convention for repeated indices.

Suppose that $\left(u, v, A_{0}, A_{1}, A_{2}\right)$ is a solution of (2.3)-(2.7). Multiplying (2.3) by $x_{k} \overline{D_{k} u}$ and integrating over $\mathbb{R}^{2}$, we obtain

$$
\begin{aligned}
\int_{\mathbb{R}^{2}}\left(\omega_{1}+A_{0}\right) u x_{k} \overline{D_{k} u} d x-\int_{\mathbb{R}^{2}} D_{j} D_{j} u x_{k} \overline{D_{k} u} d x \\
\quad-\int_{\mathbb{R}^{2}}\left(F^{\prime}\left(|u|^{2}\right) u+\partial_{s_{1}} G\left(|u|^{2},|v|^{2}\right) v\right) x_{k} \overline{D_{k} u} d x=0 .
\end{aligned}
$$

Now we set

$$
\begin{aligned}
\mathrm{I} & =\int_{\mathbb{R}^{2}}\left(\omega_{1}+A_{0}\right) u x_{k} \overline{D_{k} u} d x, \\
\mathrm{II} & =\int_{\mathbb{R}^{2}} D_{j} D_{j} u x_{k} \overline{D_{k} u} d x, \\
\mathrm{III} & =\int_{\mathbb{R}^{2}}\left(F^{\prime}\left(|u|^{2}\right) u+\partial_{s_{1}} G\left(|u|^{2},|v|^{2}\right) v\right) x_{k} \overline{D_{k} u} d x .
\end{aligned}
$$


Integrating by parts and taking real parts, we have

$$
\begin{aligned}
\operatorname{Re}\{\mathrm{I}\} & =-\int_{\mathbb{R}^{2}}\left(\omega_{1}+A_{0}\right)|u|^{2}+\frac{1}{2}|u|^{2} x_{j} \partial_{j} A_{0} d x, \\
\operatorname{Re}\{\mathrm{III}\} & =-\int_{\mathbb{R}^{2}} F\left(|u|^{2}\right)-\frac{1}{2} \partial_{s_{1}} G\left(|u|^{2},|v|^{2}\right) x_{j} \partial_{j}|u|^{2} d x .
\end{aligned}
$$

For II, we define $F_{j k}:=\partial_{j} A_{k}-\partial_{k} A_{j}$. Then we have, integrating by parts,

$$
\begin{aligned}
\mathrm{II} & =-\int_{\mathbb{R}^{2}}\left|D_{j} u\right|^{2}+x_{k} D_{j} u \overline{D_{j} D_{k} u} d x \\
& =-\int_{\mathbb{R}^{2}}\left|D_{j} u\right|^{2}+x_{k} D_{j} u \overline{\left(D_{k} D_{j} u+i F_{j k} u\right)} d x,
\end{aligned}
$$

where we used the identity $D_{j} D_{k} u=D_{k} D_{j} u+i F_{j k} u$. Taking the real part, we obtain

$$
\begin{aligned}
\operatorname{Re}\{\mathrm{II}\} & =-\int_{\mathbb{R}^{2}}\left|D_{j} u\right|^{2}+\frac{1}{2} x_{k} \partial_{k}\left(\left|D_{j} u\right|^{2}\right)+x_{k} F_{j k} \operatorname{Im}\left(\bar{u} D_{j} u\right) d x \\
& =-\int_{\mathbb{R}^{2}} x_{k} F_{j k} \operatorname{Im}\left(\bar{u} D_{j} u\right) d x .
\end{aligned}
$$

Multiplying (2.4) by $x_{k} \overline{D_{k} v}$ and integrating over $\mathbb{R}^{2}$, we obtain

$$
\begin{aligned}
\int_{\mathbb{R}^{2}}\left(\omega_{2}+A_{0}\right) v x_{k} \overline{D_{k} v} & d x-\int_{\mathbb{R}^{2}} D_{j} D_{j} v x_{k} \overline{D_{k} v} d x \\
& -\int_{\mathbb{R}^{2}}\left(\partial_{s_{2}} G\left(|u|^{2},|v|^{2}\right) v-F_{12} v\right) x_{k} \overline{D_{k} v} d x=0 .
\end{aligned}
$$

Now we set

$$
\begin{aligned}
& \mathrm{IV}=\int_{\mathbb{R}^{2}}\left(\omega_{2}+A_{0}\right) v x_{k} \overline{D_{k} v} d x, \quad \mathrm{~V}=\int_{\mathbb{R}^{2}} D_{j} D_{j} v x_{k} \overline{D_{k} v} d x, \\
& \mathrm{VI}=\int_{\mathbb{R}^{2}}\left(\partial_{s_{2}} G\left(|u|^{2},|v|^{2}\right) v-F_{12} v\right) x_{k} \overline{D_{k} v} d x .
\end{aligned}
$$

Integrating by parts and taking real parts, we have

$$
\begin{aligned}
\operatorname{Re}\{\mathrm{VI}\} & =-\int_{\mathbb{R}^{2}}\left(\omega_{2}+A_{0}\right)|v|^{2}+\frac{1}{2}|v|^{2} x_{j} \partial_{j} A_{0} d x, \\
\operatorname{Re}\{\mathrm{V}\} & =-\int_{\mathbb{R}^{2}} x_{k} F_{j k} \operatorname{Im}\left(\bar{v} D_{j} v\right) d x, \\
\operatorname{Re}\{\mathrm{VI}\} & =-\int_{\mathbb{R}^{2}} \frac{1}{2} F_{12} x_{j} \partial_{j}|v|^{2}-\frac{1}{2} \partial_{s_{2}} G\left(|u|^{2},|v|^{2}\right) x_{j} \partial_{j}|v|^{2} d x
\end{aligned}
$$

We put (2.10), (2.11) and (2.13) in the equations (2.9) and (2.12). Taking equations (2.5)-(2.7) into accout, we obtain

$$
\int_{\mathbb{R}^{2}} F\left(|u|^{2}\right)+G\left(|u|^{2},|v|^{2}\right)-\left(\omega_{1}+A_{0}\right)|u|^{2}-\left(\omega_{2}+A_{0}\right)|v|^{2} d x=0 .
$$

On the other hand, multiplying (2.3) and (2.4) by $\bar{u}$ and $\bar{v}$ respectively and integrating them, we derive 


$$
\begin{aligned}
\int_{\mathbb{R}^{2}}\left(\omega_{1}+A_{0}\right)|u|^{2}+\left(\omega_{2}+A_{0}\right)|v|^{2}+\left|D_{j} u\right|^{2}+\left|D_{j} v\right|^{2}+F_{12}|v|^{2} \\
\quad-F^{\prime}\left(\left|u^{2}\right|\right)|u|^{2}-\partial_{s_{1}} G\left(|u|^{2},|v|^{2}\right)|u|^{2}-\partial_{s_{2}} G\left(|u|^{2},|v|^{2}\right)|v|^{2} d x=0 .
\end{aligned}
$$

Combining (2.14) and (2.15), we conclude that

$$
\begin{aligned}
& \int_{\mathbb{R}^{2}}\left|D_{j} u\right|^{2}+\left|D_{j} v\right|^{2}+F_{12}|v|^{2}+F\left(|u|^{2}\right)-F^{\prime}\left(\left|u^{2}\right|\right)|u|^{2} \\
& \quad+G\left(|u|^{2},|v|^{2}\right)-\partial_{s_{1}} G\left(|u|^{2},|v|^{2}\right)|u|^{2}-\partial_{s_{2}} G\left(|u|^{2},|v|^{2}\right)|v|^{2} d x=0 .
\end{aligned}
$$

Now we apply Bogomol'nyi trick. Note the following identities hold

$$
\begin{aligned}
& \left|D_{j} u\right|^{2}=\left|\left(D_{1}+i D_{2}\right) u\right|^{2}+\nabla \times J_{B}-F_{12}|u|^{2}, \\
& \left|D_{j} v\right|^{2}=\left|\left(D_{1}+i D_{2}\right) v\right|^{2}+\nabla \times J_{F}-F_{12}|v|^{2}+\frac{1}{2} \Delta|v|^{2},
\end{aligned}
$$

where we denote

$$
\begin{aligned}
& J_{B}=\left(\operatorname{Im}\left(\bar{u} D_{1} u\right), \operatorname{Im}\left(\bar{u} D_{2} u\right)\right), \\
& J_{F}=\left(\operatorname{Im}\left(\bar{v} D_{1} v\right)+\frac{1}{2} \partial_{2}|v|^{2}, \operatorname{Im}\left(\bar{v} D_{2} v\right)-\frac{1}{2} \partial_{1}|v|^{2}\right),
\end{aligned}
$$

and use the notation $\nabla \times J=\partial_{1} J_{2}-\partial_{2} J_{1}$ for a vector $J=\left(J_{1}, J_{2}\right)$. Then the equation (2.16), considering the equation (2.7) and the divergence theorem, gives

$$
\begin{gathered}
\int_{\mathbb{R}^{2}}\left|\left(D_{1}+i D_{2}\right) u\right|^{2}+\left|\left(D_{1}+i D_{2}\right) v\right|^{2}+\frac{1}{2}|u|^{4}+F\left(|u|^{2}\right)-F^{\prime}\left(|u|^{2}\right)|u|^{2}+\frac{1}{2}|u|^{2}|v|^{2} \\
+G\left(|u|^{2},|v|^{2}\right)-\partial_{s_{1}} G\left(|u|^{2},|v|^{2}\right)|u|^{2}-\partial_{s_{2}} G\left(|u|^{2},|v|^{2}\right)|v|^{2} d x=0 .
\end{gathered}
$$

This completes the proof of Proposition 2.1.

\section{Statements for nonexistence results to (2.1)}

We state in this section some structural condition on $F$ and $G$ so that (2.1) only admits trivial standing wave solutions. This is an extension of the result in [5] where Jackiw-Pi model is considered. We also refer to [3] in which the nonexistence of nontrivial solutions to another field equation of Schrödinger type, the nonlinear Schrödinger-Maxwell system

$$
\left\{\begin{array}{l}
-\Delta u+V(x) u+\phi u=|u|^{p-2} u, \\
-\Delta \phi=u^{2}
\end{array}\right.
$$

is studied. It is shown in [3] that if $V$ is positive constant and $p \in(0,2) \cap(6, \infty)$, then (3.1) does not admit any nontrivial solutions $(u, \phi)$ with finite energy. When $p=6$ and $V$ is nonconstant, a nonexistence result is obtained in [1] by imposing some conditions on $V$. The above two papers also make use of Pohozaev and Nehari identities. 
Now, we state the result. Recall that we are already assuming the condition (2.2).

TheOrem 3.1. Suppose that $\left(u, v, A_{0}, A_{1}, A_{2}\right)$ is a smooth solution of the equations (2.3)-(2.7) satisfying:

(a) $u, v \in H^{1}\left(\mathbb{R}^{2}\right)$,

(b) $A_{0} \in L^{p}\left(\mathbb{R}^{2}\right)$ for some $1<p \leq \infty$,

(c) $A_{1}, A_{2} \in L^{q}\left(\mathbb{R}^{2}\right)$ for some $2<q \leq \infty$,

(d) $F\left(|u|^{2}\right), F^{\prime}\left(|u|^{2}\right)|u|^{2}, G\left(|u|^{2},|v|^{2}\right), \partial_{s_{1}} G\left(|u|^{2},|v|^{2}\right)|v|^{2}, \partial_{s_{2}} G\left(|u|^{2},|v|^{2}\right)|v|^{2}$ in $L^{1}\left(\mathbb{R}^{2}\right)$.

Assume that

$$
\left\{\begin{array}{lr}
\frac{1}{2} s^{2}+F(s)-F^{\prime}(s) s>0 & \text { for all } s>0, \\
\frac{1}{2} s_{1} s_{2}+G\left(s_{1}, s_{2}\right)-\partial_{s_{1}} G\left(s_{1}, s_{2}\right) s_{1}-\partial_{s_{2}} G\left(s_{1}, s_{2}\right) s_{2} & \geq 0 \\
& \text { for all } s_{1}, s_{2}>0 .
\end{array}\right.
$$

Then we have that $u \equiv 0, A_{0}$ is a real constant and

$$
\left(D_{1}+i D_{2}\right) v=0, \quad \partial_{1} A_{2}-\partial_{2} A_{1}=-\frac{1}{2}|v|^{2} .
$$

Moreover, we also have $v \equiv 0$ if $A_{0} \neq-\omega_{2}$.

Note that Theorem 3.1 immediately implies Theorem 1.2. We will give a proof of Theorem 3.1 in Section 4.

Now, we switch the strict inequality sign in the condition (3.2). Then we have the following result, which will be proved in Section 4 .

Theorem 3.2. Suppose that $\left(\phi, \psi, A_{0}, A_{1}, A_{2}\right)$ is a smooth solution of the equations (2.3)-(2.7) satisfying (a)-(d) of Theorem 3.1. Assume that

$$
\left\{\begin{array}{lr}
\frac{1}{2} s^{2}+F(s)-F^{\prime}(s) s \geq 0 & \text { for all } s>0, \\
\frac{1}{2} s_{1} s_{2}+G\left(s_{1}, s_{2}\right)-\partial_{s_{1}} G\left(s_{1}, s_{2}\right) s_{1}-\partial_{s_{2}} G\left(s_{1}, s_{2}\right) s_{2} & >0 \\
& \text { for all } s_{1}, s_{2}>0 .
\end{array}\right.
$$

Then one of the following holds:

(a) $u \equiv 0, v \equiv 0, A_{0}$ is a real constant and $\partial_{1} A_{2}-\partial_{2} A_{1}=0$;

(b) $u \equiv 0, A_{0}=-\omega_{2}, v \not \equiv 0$ and

$$
\left(D_{1}+i D_{2}\right) v=0, \quad \partial_{1} A_{2}-\partial_{2} A_{1}=-\frac{1}{2}|v|^{2} ;
$$

(c) $v \equiv 0, A_{0}=|u|^{2} / 2-\omega_{1}, u \neq \equiv 0$ and

$$
\begin{gathered}
\left(D_{1}+i D_{2}\right) u=0, \quad \partial_{1} A_{2}-\partial_{2} A_{1}=-\frac{1}{2}|u|^{2}, \quad F(s)=\frac{1}{2} s^{2} \quad \text { for } s \in[0, T], \\
\text { where } T=\max _{x \in \mathbb{R}^{2}}|u(x)|^{2} .
\end{gathered}
$$


It is easy to see that Theorem 3.2 implies both of Theorem 1.3 and the following nonexistence results.

Corollary 3.3. Let $\left(\phi, \psi, A_{0}, A_{1}, A_{2}\right)$ be a solution of the equations (2.3)(2.7) satisfying (a)-(d) of Theorem 3.2. Suppose that $F$ and $G$ satisfy the condition (3.3) and there is a sequence $\left\{s_{n}\right\} \rightarrow 0$ such that $F\left(s_{n}\right) \neq s_{n}^{2} / 2$ for all $n$. Suppose also that $A_{0} \neq-\omega_{2}$. Then one has $\phi \equiv 0$ and $\psi \equiv 0$.

\section{Proof of Theorems 1.1, 3.1 and 3.2}

We can easily prove all the theorems by using identity (2.8).

Proof of Theorem 1.1. Set $\omega_{1}=\omega_{2}=0, F(s)=s^{2} / 2$ and $G\left(s_{1}, s_{2}\right)=$ $s_{1} s_{2} / 2$. Then Proposition 2.1 implies $\left(D_{1}+i D_{2}\right) \phi=0$ and $\left(D_{1}+i D_{2}\right) \psi=0$.

It remains to show $A_{0}=|\phi|^{2} / 2$ to complete the proof of Theorem 1.1.

From the equation (1.3) and the factorization identity (1.8), we can check that the equation $A_{0} \phi=|\phi|^{2} \phi / 2$ is satisfied. Also $\nabla A_{0}=\nabla|\phi|^{2} / 2$ from equations (1.5) and (1.6) so that $A_{0}=|\phi|^{2} / 2+c$ for some $c \in \mathbb{R}$. Thus, we have $c \phi \equiv 0$ and conclude that $c=0$ since $\phi \not \equiv 0$.

Proof of Theorem 3.1. Observe that the condition (3.2) and Proposition 2.1 imply that

$$
u \equiv 0 \quad \text { and } \quad\left(D_{1}+i D_{2}\right) v=0 .
$$

Then, equations (2.5)-(2.6) become $\nabla A_{0}=0$ so that $A_{0}$ is a real constant. The equation (2.7) becomes $\partial_{1} A_{2}-\partial_{2} A_{1}=-|v|^{2} / 2$. From the factorization identity (1.8) and the condition (2.2), we see that the equation (2.4) becomes $\left(\omega_{2}+A_{0}\right) v=0$. Therefore $v \equiv 0$ if $A_{0} \neq-\omega_{2}$.

Proof of Theorem 3.2. We deduce from the condition (3.3) and Proposition 2.1 that

$$
u v \equiv 0, \quad\left(D_{1}+i D_{2}\right) u=0, \quad\left(D_{1}+i D_{2}\right) v=0 .
$$

Then we have

$$
\begin{array}{ll}
\left(\left(\omega_{1}+A_{0}\right)+\frac{1}{2}|u|^{2}-F^{\prime}\left(|u|^{2}\right)\right) u=0, & \left(\omega_{2}+A_{0}\right) v=0 \\
A_{0}=\frac{1}{2}|u|^{2}+c, & F_{12}=-\frac{1}{2}\left(|u|^{2}+|v|^{2}\right) .
\end{array}
$$

for some $c \in \mathbb{R}$.

Now we introduce the following lemma and refer to Proposition 5.1 in [8] for the proof.

Lemma 4.1. Let $f$ be a nonzero smooth complex function and $A_{1}, A_{2}$ be smooth real functions on $\mathbb{R}^{2}$ such that $\left(D_{1}+i D_{2}\right) f=0$. Then the zero set of $f$ has empty interior. 
Considering (4.1) and Lemma 4.1, we have the following three cases:

Case 1. Suppose that $u \equiv 0$ and $v \equiv 0$. Then we can derive the conclusion (a) of Theorem 3.2 from (4.2).

Case 2. Suppose that $v \not \equiv 0$. Then $u \equiv 0$ by Lemma 4.1 and the conclusion (b) of Theorem 3.2 is also immediate from (4.2).

Case 3. Suppose that $u \not \equiv 0$. Then $v \equiv 0$ and $\omega_{1}+c+|u|^{2}-F^{\prime}\left(|u|^{2}\right)=0$ by (4.2). Taking limit $|x| \rightarrow \infty$, we see that $c=-\omega_{1}$ and $|u|^{2}-F^{\prime}\left(|u|^{2}\right) \equiv 0$. Therefore we have $A_{0}=|u|^{2} / 2-\omega_{1}$ and $s-F^{\prime}(s)=0$ on $s \in\left[0, \max _{x \in \mathbb{R}^{2}}|u(x)|^{2}\right]$. This completes the proof of Theorem 3.2.

Acknowledgements. H. Huh was supported by Basic Science Research Program through the National Research Foundation of Korea (NRF) funded by the Ministry of Education, Science and Technology (2011-0015866).

\section{REFERENCES}

[1] A. Azzollini And A. Pomponio, Ground state solutions for the nonlinear SchrodingerMaxwell equations, J. Math. Anal. Appl. 345 (2008), no. 1, 90-108.

[2] L.C. Evans, Partial differential equations, Second edition, Graduate Studies in Mathematics 19, 2010.

[3] T. D'Aprile and D. Mugnai, Non-existence results for the coupled Klein-Gordon-Maxwell equations, Adv. Nonlinear Stud. 4 (2004), no. 3, 307-322.

[4] H. Huh And J. Seok, The equivalence of the Chern-Simons-Schrödinger equations and its self-dual system, J. Math. Phys. 54 (2013), 021502, 5 pp.

[5] H. HuH, Nonexistence results of semilinear elliptic equations coupled with the ChernSimons gauge field, Abstr. Appl. Anal. (2013), Article ID 467985, 5 pages.

[6] R. JaCkiw And S.-Y. PI, Classical and quantal nonrelativistic Chern-Simons theory, Phys. Rev. D. 42 (1990), 3500-3513.

[7] M. Leblanc, G. Lozano and H. Min, Extended superconformal Galilean symmetry in Chern-Simons matter systems, Ann. Physics 219 (1992), no. 2, 328-348.

[8] C.H. TAubes, Vortices and monopoles, Birkhäuser, Boston, (1980).

HyungJin HuH

Department of Mathematics

Chung-Ang University

Seoul, 156-756, REPUBLIC of KOREA

E-mail address: huh@cau.ac.kr

JinMYOUng SEOK

Department of Mathematics

Kyonggi University

Suwon, 443-760, REPUBLIC of KOREA

E-mail address: jmseok@kgu.ac.kr

TMNA : Volume $46-2015-\mathrm{N}^{\mathrm{O}} 1$ 